\title{
A evolução da citação na Revista Portuguesa de Medicina Geral e Familiar: de 2000-2008 para 2009-2015
}

Carolina Henriques Abreu, ${ }^{1}$ Inês Rosendo, ${ }^{2}$ Luiz Miguel Santiago ${ }^{3}$

\section{RESUMO}

Objetivos: Medir a citação bibliográfica de artigos já publicados na Revista Portuguesa de Medicina Geral e Familiar (RPMGF), comparando com os anteriores valores de citação.

Tipo de estudo: Estudo observacional, transversal e descritivo.

Local: Faculdade de Medicina da Universidade de Coimbra.

Material: Todos os números da RPMGF publicados entre 2009 e 2015 (inclusive).

Métodos: Revisão sistemática quanto ao número total de referências bibliográficas, número de referências a artigos publicados em Portugal e número de referências bibliográficas de artigos já publicados na RPMGF e constante do seu sítio na Internet. Comparação com os resultados de anterior trabalho e sua comparação com os anteriores.

Resultados: O número total de referências nos artigos publicados foi de $12,0 \pm 11,2$, as de artigos Portugueses de $2,4 \pm 3,0$ e as de artigos publicados nesta RPMGF de $0,6 \pm 1,2$. As dinâmicas de crescimento relativamente aos resultados anteriores são negativas para referências da revista $(-33,3)$, para referências portuguesas $(-14,3)$ e para referências totais $(-28,6)$.

Conclusões: Os valores de citação encontrados são baixos e com dinâmicas de crescimento negativas em relação aos já anteriormente publicados.

Palavras-chave: Referenciação bibliográfica; Análise de citações; Revista científica; RPMGF.

\section{INTRODUÇÃO}

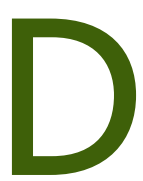

esde a sua primeira publicação em janeiro de 1984 que a então designada Revista Portuguesa de Clínica Geral tem seguido e influenciado o desenvolvimento da medicina geral e familiar (MGF) em Portugal. ${ }^{1}$ No decorrer das últimas três décadas, a agora denominada Revista Portuguesa de Medicina Gerale Familiar (Revista) ${ }^{2}$ evoluiu no sentido de acompanhar o conhecimento médico e desenvolvimento tecnológico, afirmando-se atualmente como a publicação mais prestigiada da área em Portugal. $^{3}$

1. Estudante de $6^{\circ}$ ano do Mestrado Integrado em Medicina. Faculdade de Medicina da Universidade de Coimbra.

2. Médica Assistente em Medicina Geral e Familiar. ARS Centro. Assistente convidada da Faculdade de Medicina da Universidade de Coimbra.

3. Médico Especialista em Medicina Geral e Familiar. USF Topázio. Professor Associado Convidado da Faculdade de Medicina da Universidade de Coimbra.
Pela sua importância, a Revista tem sido objeto de alguns estudos de análise de citações, ${ }^{4-5}$ bem como de avaliação da gestão de artigos por parte do corpo editorial, ${ }^{6}$ tendo revelado existir uma preferência dos autores pela citação da Revista. ${ }^{5}$ Contudo, num estudo de 2008, os valores de citação de artigos da Revista mostraram-se baixos $(0,9 \pm 1,7$ citações em média, entre 2000 e 2006). ${ }^{4}$

Ao longo dos anos, os diferentes corpos editoriais da Revista têm dado passos significativos no sentido de trazer aos leitores uma revista de elevada qualidade, sendo exemplo disso a disponibilização gratuita online desde 2000 e a indexação à plataforma SciELO Portugal desde 2011, permitindo que as suas publicações ganhem mais impacto e visibilidade, para que os artigos da revista sejam mais vezes citados, comentados e discutidos. ${ }^{7}$ 
Em 2005, a Revista Portuguesa de Clínica Geral era considerada como a revista médica de língua portuguesa mais lida com regularidade em Portugal., ${ }^{4,-9}$ Mas a métrica de avaliação passa mais pela contagem do número de vezes que um artigo é citado do que pelo número total de leitores. Importa conhecer objetivamente a progressão e o panorama atual desta realidade, cuja relevância foi assumida publicamente por várias direções editoriais, ${ }^{4,8-9}$ apontando sugestões consensualizadas de melhoria.

Este estudo pretende medir a citação bibliográfica da Revista Portuguesa de Medicina Geral e Familiar, na continuação de um artigo anteriormente publicado nesta Revista. ${ }^{4}$ Especificamente pretende-se estudar o número total de referências bibliográficas, o número de referências bibliográficas de artigos publicados em revistas portuguesas e o número de autorreferências bibliográficas da Revista, em função do número total de referências em cada artigo, nos números publicados no período de 2009 a 2015 (inclusive). Pretende-se ainda comparar os atuais níveis de citação com os anteriormente obtidos em 2008, numa perspetiva de evolução temporal. ${ }^{4}$

\section{MÉTODOS}

Elaborou-se um trabalho observacional e descritivo. Foi realizada a revisão sistemática de todos os artigos de todos os números da Revista entre 2009 e 2015, ambos os anos inclusive, elaborada a partir do sítio Internet da Revista (www.rpmgf.pt), incidindo sobre o número de artigos, o número total de referências bibliográficas, o número de referências bibliográficas de artigos publicados em Portugal e o número de referências bibliográficas de artigos já publicados na Revista. $^{10}$

Na tentativa de melhor explorar o panorama de citação da Revista foram aplicados os indicadores de citação já aplicados em anterior artigo: ${ }^{4}$

- Indicador 1: Número de cita- ções de artigos publicados na Revista / Número total de citações bibliográficas na Revista.

- Indicador 2: Número de citações de artigos publicados na Revista / Número total de citações bibliográficas portuguesas na Revista.

- Indicador 3: Por ano: Número de citações de artigos publicados na Revista / Número de artigos publicados na Revista.

- Indicador 4: Número total de citações bibliográficas portuguesas na Revista / Número total de citações bibliográficas na Revista.

Foi realizada a comparação dos resultados do presente estudo com os resultados previamente obtidos no trabalho publicado em 2008. ${ }^{4}$

Para isso foi avaliada a dinâmica de crescimento $(\Delta)$ entre os valores médios de citação do estudo atual e anterior, bem como entre os indicadores de citação aplicados.

\section{RESULTADOS}

No presente estudo, entre 2009 e 2015, verificou-se que a Revista publicou 630 artigos, nas várias categorias que detém. O Quadro I mostra a distribuição dos

\begin{tabular}{|c|c|c|c|c|c|c|c|c|}
\hline Tipo / Ano & $\begin{array}{c}2009 \\
(\%)\end{array}$ & $\begin{array}{c}2010 \\
(\%)\end{array}$ & $\begin{array}{c}2011 \\
(\%)\end{array}$ & $\begin{array}{c}2012 \\
(\%)\end{array}$ & $\begin{array}{c}2013 \\
(\%)\end{array}$ & $\begin{array}{c}2014 \\
(\%)\end{array}$ & $\begin{array}{c}2015 \\
(\%)\end{array}$ & $\begin{array}{l}\text { Total } \\
\text { (\%) }\end{array}$ \\
\hline Clube Leitura & 16,9 & 11,4 & 19,6 & 27,2 & 23,4 & 22,5 & 20,8 & 19,7 \\
\hline Editorial & 6,8 & 9,5 & 17,5 & 21,0 & 20,8 & 21,3 & 23,6 & 16,2 \\
\hline Original & 10,2 & 6,7 & 16,5 & 18,5 & 16,9 & 15,0 & 13,9 & 13,5 \\
\hline Dossier & 31,4 & 14,3 & 9,3 & 0,0 & 0,0 & 0,0 & 0,0 & 9,7 \\
\hline POEM & 11,9 & 27,6 & 5,2 & 1,2 & 1,3 & 0,0 & 1,4 & 8,1 \\
\hline Revisão & 7,6 & 6,7 & 6,2 & 7,4 & 7,8 & 7,5 & 4,2 & 6,8 \\
\hline Artigo breve & 0,8 & 0,0 & 6,2 & 6,2 & 6,5 & 8,8 & 16,7 & 5,7 \\
\hline Relato de Caso & 2,5 & 1,9 & 4,1 & 7,4 & 7,8 & 7,5 & 9,7 & 5,4 \\
\hline Carta ao Diretor & 0,0 & 3,8 & 4,1 & 4,9 & 9,1 & 10,0 & 5,6 & 4,9 \\
\hline WebSaúde & 9,3 & 11,4 & 6,2 & 0,0 & 0,0 & 0,0 & 0,0 & 4,6 \\
\hline Opinião & 2,5 & 4,8 & 2,1 & 3,7 & 5,2 & 6,3 & 2,8 & 3,8 \\
\hline Prática & 0,0 & 1,0 & 1,0 & 1,2 & 1,3 & 1,3 & 0,0 & 0,8 \\
\hline Formação & 0,0 & 1,0 & 1,0 & 1,2 & 0,0 & 0,0 & 0,0 & 0,5 \\
\hline Documentos & 0,0 & 0,0 & 1,0 & 0,0 & 0,0 & 0,0 & 1,4 & 0,3 \\
\hline Total (número) & 118 & 105 & 97 & 81 & 77 & 80 & 72 & 630 \\
\hline
\end{tabular}




\begin{tabular}{|c|c|c|c|}
\hline \multicolumn{4}{|c|}{$\begin{array}{l}\text { QUADRO II. Média de referências bibliográficas por tipo de } \\
\text { artigo ao longo do período considerado }\end{array}$} \\
\hline Tipo de artigo & $\begin{array}{l}\text { Referências } \\
\text { totais }\end{array}$ & $\begin{array}{l}\text { Referências } \\
\text { portuguesas }\end{array}$ & $\begin{array}{l}\text { Referências } \\
\text { da Revista }\end{array}$ \\
\hline Formação & $15,7 \pm 16,0$ & $4,0 \pm 7,0$ & $3,0 \pm 5,2$ \\
\hline Documentos & $11,0 \pm 12,7$ & $2,0 \pm 2,8$ & $1,5 \pm 2,1$ \\
\hline Prática & $20,2 \pm 13,3$ & $5,2 \pm 3,0$ & $1,6 \pm 1,7$ \\
\hline Editorial & $8,0 \pm 5,1$ & $2,6 \pm 2,2$ & $1,3 \pm 1,5$ \\
\hline Original & $21,7 \pm 9,6$ & $4,3 \pm 2,7$ & $0,9 \pm 1,4$ \\
\hline Carta ao Diretor & $4,8 \pm 4,6$ & $2,2 \pm 2,0$ & $1,1 \pm 1,1$ \\
\hline Dossier & $19,1 \pm 14,4$ & $3,6 \pm 4,4$ & $0,5 \pm 1,4$ \\
\hline Relato de Caso & $14,6 \pm 7,6$ & $2,9 \pm 2,5$ & $0,5 \pm 1,2$ \\
\hline Artigo Breve & $12,8 \pm 7,7$ & $2,2 \pm 3,7$ & $0,4 \pm 1,3$ \\
\hline Opinião & $21,5 \pm 14,4$ & $4,6 \pm 5,8$ & $0,4 \pm 1,0$ \\
\hline EM & $7,3 \pm 12,6$ & $1,0 \pm 1,9$ & $0,2 \pm 0,6$ \\
\hline Clube de Leitura & $5,4 \pm 4,1$ & $0,9 \pm 1,2$ & $0,1 \pm 0,5$ \\
\hline Revisão & $20,8 \pm 10,6$ & $1,5 \pm 1,4$ & $0,1 \pm 0,3$ \\
\hline WebSaúde & $0,0 \pm 0,0$ & $0,0 \pm 0,0$ & $0,0 \pm 0,0$ \\
\hline Média Total & $12,0 \pm 11,2$ & $2,4 \pm 3,0$ & $0,6 \pm 1,2$ \\
\hline
\end{tabular}

\begin{tabular}{|c|c|c|c|c|}
\hline \multicolumn{5}{|c|}{$\begin{array}{l}\text { QUADRO III. Referências totais, portuguesas e de artigos } \\
\text { previamente na Revista (período de 2009-2015) }\end{array}$} \\
\hline & & $\begin{array}{l}\text { Referências } \\
\text { totais }\end{array}$ & $\begin{array}{l}\text { Referências } \\
\text { portuguesas }\end{array}$ & $\begin{array}{l}\text { Referências } \\
\text { da Revista }\end{array}$ \\
\hline \multicolumn{2}{|c|}{ Média $\pm d p$} & $12,0 \pm 11,2$ & $2,4 \pm 3,0$ & $0,6 \pm 1,2$ \\
\hline \multirow{2}{*}{ IC a $5 \%$} & Superior & 12,9 & 2,6 & 0,7 \\
\hline & Inferior & 11,1 & 2,1 & 0,5 \\
\hline \multicolumn{2}{|c|}{ Mediana } & 9,0 & 1,0 & 0,0 \\
\hline
\end{tabular}

\begin{tabular}{|c|c|c|c|}
\hline & $\begin{array}{l}\text { Estudo } \\
\text { anterior }\end{array}$ & $\begin{array}{c}\text { Estudo } \\
\text { atual }\end{array}$ & $\Delta$ \\
\hline Média de referências da Revista & 0,9 & 0,6 & $-33,3 \%$ \\
\hline Média de referências portuguesas & 2,8 & 2,4 & $-14,3 \%$ \\
\hline Média de referências totais & 16,8 & 12,0 & $-28,6 \%$ \\
\hline
\end{tabular}

artigos, seu tipo e secção, por ano de publicação. O Quadro II mostra a média de referências bibliográficas por tipo de artigo ao longo do período considerado.

Relativamente aos valores de referenciação bibliográfica, encontramos no Quadro III uma média de 12 referências por artigo, com 2,4 referências de artigos publicados em Portugal e 0,6 referências da Revista.

Comparando os valores de citação com os anteriormente obtidos em 2008, o Quadro IV mostra uma dinâmica de crescimento negativa para os três tipos de referenciação em estudo, principalmente para a referenciação de artigos previamente publicados na Revista $(-33,3)$.

O resultado dos indicadores 1,2 e 4 é apresentado no QuadroV, verificando-se uma proporção de 0,05 para referências da Revista/referências totais, de 0,25 para referências da Revista/referências portuguesas e de 0,20 para referências portuguesas/referências totais. Interessa salientar que, em relação aos resultados anteriores, ${ }^{4} \mathrm{o}$ indicador 2 apresenta uma dinâmica de crescimento negativa $(-16,70)$ e o indicador 4 revela um crescimento positivo quanto à citação de artigos nacionais $(17,60)$.

No Quadro VI são apresentados, em detalhe, os valores obtidos para o indicador 3, comparando o estudo anterior e o atual, sendo de assinalar dois picos recentes de maior produção nos anos $2011 \mathrm{e}$ 2012.

\section{DISCUSSÃO}

Os valores de citação encontrados merecem reflexão. Desconhecendo-se trabalhos com este tipo de indicadores em outras revistas médicas, foi encontrado um valor de $0,05,0,25$ e 0,58 respetivamente quanto às razões das referências bibliográficas a artigos publicados na Revista sobre o total de referências, sobre o total de referências portuguesas e sobre o número de artigos entre 2009 e 2015.

Quando comparados com os resultados anteriormente obtidos em 2008, verifica-se que existe uma dinâmica de crescimento negativa para os três tipos de referências em estudo. É na citação de artigos previamente publicados na Revista que a dinâmica de crescimento é mais negativa $(-33,3 \%){ }^{4}$ Tal pode levantar várias linhas de hipóteses explicativas 
QUADRO V. Indicadores de citação - dinâmica de crescimento $(\Delta)$

\begin{tabular}{l|c|c|c} 
& Anterior & Atual & $\Delta$ \\
\hline Indicador 1 & 0,05 & 0,05 & $0,00 \%$ \\
\hline Indicador 2 & 0,30 & 0,25 & $-16,70 \%$ \\
\hline Indicador 4 & 0,17 & 0,20 & $17,60 \%$ \\
\hline
\end{tabular}

QUADRO VI. Indicador 3 de citação - dinâmica de crescimento $(\Delta)$

\begin{tabular}{l|c|c|c|c} 
Ano/Indicador & Indicador 3 & Ano/Indicador & Indicador 3 & $\Delta$ \\
\hline $\mathbf{2 0 0 0}$ & 1,00 & $\mathbf{2 0 0 9}$ & 0,47 & --- \\
\hline $\mathbf{2 0 0 1}$ & 1,70 & $\mathbf{2 0 1 0}$ & 0,44 & --- \\
\hline $\mathbf{2 0 0 2}$ & 0,86 & $\mathbf{2 0 1 1}$ & 0,76 & --- \\
\hline $\mathbf{2 0 0 3}$ & 0,27 & $\mathbf{2 0 1 2}$ & 0,73 & --- \\
\hline $\mathbf{2 0 0 4}$ & 0,52 & $\mathbf{2 0 1 3}$ & 0,57 & --- \\
\hline $\mathbf{2 0 0 5}$ & 1,18 & $\mathbf{2 0 1 4}$ & 0,60 & --- \\
\hline $\mathbf{2 0 0 6}$ & 0,84 & $\mathbf{2 0 1 5}$ & 0,58 & --- \\
\hline Total & $\mathbf{0 , 8 7}$ & Total & $\mathbf{0 , 5 8}$ & $-33,3 \%$
\end{tabular}

em maior citação na Revista, parecendo assim que os artigos publicados, por razões que deverão ser estudadas, não são alvo de citação, o que de certa forma encurta o impacto dos artigos publicados na Revista.

Parece ser necessário que os médicos de MGF invistam na leitura da Revista para suporte à realização dos seus trabalhos. Não foi objeto deste trabalho a temática dos artigos. Ainda assim, é possível pensar que alguns artigos a serem propostos tenham suporte bibliográfico em anteriores publicações da Revista.

Sugere-se a maior promoção da Revista e dos seus artigos, nomeadamente nas redes sociais, por $e$-mail e nos congressos, de modo a amplificar a sua leitura, o que posteriormente incentivará os clínicos na valorização dos artigos da Revista para suporte aos seus trabalhos. Igualmente a inclusão da Revista no ensino pré-graduado, assim como no pós-graduado, podem ser fatores de crescimento da citação que, de momen-

como a da relativa pouca importância dada aos artigos da RPMGF por esta não estar indexada na PubMed/MEDLINE ou pela temática pouco apelativa aos leitores, surgindo a necessidade de realizar este trabalho explicativo.

Ainda assim, a razão entre referências portuguesas/referências totais apresenta uma dinâmica de crescimento positiva $(+17,6 \%)$, o que indica uma maior tendência para citar artigos nacionais aquando da realização dos trabalhos.

Estes resultados parecem ser insatisfatórios até porque, aquando do anterior trabalho, a situação foi exposta na própria Revista e em Congresso Nacional de Medicina Geral e Familiar e foram feitas várias sugestões no sentido de consciencializar os clínicos, incentivando à mudança. No entanto, pode constatar-se que nem todas essas sugestões/intervenções foram bem-sucedidas, sendo exemplo disso a ausência de indexação da Revista à PubMed. Ainda assim, foi feita a indexação na SciELO Portugal e melhorado o sítio da Revista, que permite agora o acesso a todos os artigos. Admitindo um incremento no acesso ao sítio da Revista e, subsequentemente, na leitura, tal não parece redundar to, tem valores negativos na dinâmica entre os dois períodos temporais estudados.

Sugere-se a discussão e monitorização futura desta realidade.

\section{REFERÊNCIAS BIBLIOGRÁFICAS}

1. Pisco L. Uma nova imagem, os mesmos princípios [A new image, the same principles]. Rev Port Clin Geral. 2000;16(1):7-8. Portuguese

2. Braga R. Um outro nome para uma realidade em mudança [Another name for a changing reality]. Rev Port Clin Geral. 2011;27(6):495-6. Portuguese

3. Broeiro P. 30 Anos de RPMGF como organização aprendente [Thirty years' experience with the RPMGF as a learning organization]. Rev Port Med Geral Fam. 2014;30(2):71-2. Portuguese

4. Rosendo I, Santos T, Martins D, Pimenta G, Neto MG, Francisco MP, et al. A citação da Revista Portuguesa de Clínica Geral na Revista Portuguesa de Clínica Geral [Citation of articles from the Portuguese Journal of General Practice in the Portuguese Journal of General Practice]. Rev Port Clin Geral. 2008;24(4):457-61. Portuguese

5. Antunes ML. Citações nos artigos publicados na revista portuguesa de clínica geral: análise da década 2000-2009 [Citations in articles published in the Portuguese Journal of General Practice: analysis of the decade 2000-2009]. Rev Port Med Geral Fam. 2011;27(3):265-72. Portuguese

6. Pinto D. Gestão dos manuscritos submetidos à Revista Portuguesa de Medicina Geral e Familiar nos últimos cinco anos [The handling of ma- 
nuscripts submitted to the Portuguese Journal of Family Medicine and General Practice in the past five years]. Rev Port Med Geral Fam. 2013; 29(4):221-3. Portuguese

7. Braga R. Uma nova página [A new page]. Rev Port Med Geral Fam. 2011; 27(2):137-8. Portuguese

8. Sousa JC, Mateus A. Revistas médicas e formação em medicina geral e familiar: hábitos e necessidades de leitura de internos e de orientadores de formação do internato complementar [Medical journals and training in family medicine]. Rev Port Clin Geral. 2005;21(1):23-42. Portuguese

9. Braga R. Investigação e publicação científica em medicina geral e familiar. In: Publicação científica: motivação e formação (workshop) - $1^{\text {a }}$ Jornadas do Centro de Neurociências da CUF Porto. Porto: Hospital CUF Porto; 2016. Available from: http://www.actamedicaportuguesa.com/ info/apresentacoes_simposio/09-Raquel\%20Braga_I-Simp.pdf

10. Revista Portuguesa de Medicina Geral e Familiar. rpmgf.pt [homepa- ge]. Lisboa: Associação Portuguesa de Medicina Geral e Familiar. Available from: http://www.rpmgf.pt/ojs/index.php/rpmgf

\section{CONFLITOS DE INTERESSE}

Os autores Carolina Henriques Abreu e Luiz Miguel Santiago declaram não possuir quaisquer conflitos de interesse.

A autora Inês Rosendo declara ter integrado a equipa editorial da Revista Portuguesa de Medicina Geral e Familiar entre 2000 e 2014.

\section{ENDEREÇO PARA CORRESPONDÊNCIA}

Carolina Henriques Abreu

E-mail: carolina429535@gmail.com

Recebido em 10-04-2016

Aceite para publicação em 04-12-2017

\section{ABSTRACT}

\section{EVOLUTION OF THE CITATION OF THE PORTUGUESE JOURNAL OF GENERAL PRACTICE}

Objetive: To measure the bibliographic citation of articles previously published in the Portuguese Journal of General Practice and compare these results with previous citation values.

Study design: Observational, transversal and descriptive study.

Place: Faculty of Medicine, University of Coimbra, Portugal.

Population: All issues of the Portuguese Journal of General Practice published between 2009 and 2015 (including).

Methods: Systematic review of the total number of bibliographic references, number of bibliographic references in articles published in Portugal and number of bibliographic references in articles published in this journal and available on its website. Comparison with previously published results.

Results: Total references were $12.0 \pm 11.2$ in published articles, $2.4 \pm 3.0$ in articles published in Portuguese journals and $0.6 \pm 1.2$ in articles published in this journal. The growth dynamics with respect to previous results are negative for the journal's references (-33.3), for Portuguese references (-14.3), and for total references (-28.6).

Conclusions: The citation values found are low and with negative growth dynamics in relation to those previously published.

Keywords: Bibliographic citation; Citation analysis; Scientific Journal; RPMGF. 